\title{
Donor-specific cell-based assays in studying sensitivity to low-dose radiation: a population-based perspective
}

\author{
Dora II'yasova $^{1}$ *, Alexander Kinev ${ }^{2}$, C. David Melton ${ }^{1}$ and Faith G. Davis ${ }^{3}$ \\ ${ }^{1}$ Division of Epidemiology and Biostatistics, School of Public Health, Georgia State University, Atlanta, GA, USA \\ ${ }^{2}$ Creative Scientist, Inc., Durham, NC, USA \\ ${ }^{3}$ School of Public Health, University of Alberta, Edmonton, AB, Canada
}

\section{Edited by:}

Jimmy Thomas Efird, ECU Heart

Institute, Brody School of Medicine,

USA

\section{Reviewed by:}

Wilfried Joachim Juergen Karmaus,

University of Memphis, USA

Eugenia M. Bastos, Bastos

Consulting, USA

\section{*Correspondence:}

Dora Il'yasova, School of Public

Health, Georgia State University,

Urban Life Building, Suite 878, 140

Decatur Street, PO Box 3995, Atlanta,

GA 30303, USA

e-mail: dilyasova@gsu.edu
Currently, a linear no-threshold model is used to estimate health risks associated with exposure to low-dose radiation, a prevalent exposure in the general population, because the direct estimation from epidemiological studies suffers from uncertainty. This model has been criticized based on unique biology of low-dose radiation. Whether the departure from linearity is toward increased or decreased risk is intensely debated. We present an approach based on individual radiosensitivity testing and discuss how individual radiosensitivity can be assessed with the goal to develop a quantifiable measure of cellular response that can be conducted via high-throughput population testing.

Keywords: low-dose ionizing radiation, individual variability, biomarkers, epidemiology

\section{WHY DO WE NEED A NEW APPROACH?}

High-dose ionizing radiation ( $>1 \mathrm{~Gy}$ ) is an established human carcinogen, with a linear relationship to cancer risk $(1,2)$. Its major mechanism of carcinogenicity is DNA damage $(1,2)$. In contrast, risk estimates from epidemiological studies examining low radiation doses $(<0.2 \mathrm{~Gy})$ suffer from a significant uncertainty $(3,4)$. The biological effects of low-dose radiation differ from those observed at high doses and are mostly unrelated to DNA damage per se (5-13). In the absence of reliable estimates, the conventional approach extrapolates the linear trend observed at high-dose radiation to low-dose exposures, which is known as the "linear no-threshold hypothesis" (LNTH) $(1,14-17)$. Such extrapolation has been criticized, because it assumes the same carcinogenic mechanism as operating at high doses, whereas the biological phenomena are specific to low-dose exposure. These effects can be summarized as three competing hypotheses. The bystander effect hypothesis predicts a risk greater than expected according to the LNTH, because irradiated cells can transmit radiation effects to un-irradiated neighboring cells $(9-13,16,18,19)$. The threshold hypothesis considers radiation-induced cancer risk at low doses to be close to zero due to a threshold of radiation-induced damaging effects (17, 20, 21). Finally, the adaptive response hypothesis considers a beneficial effect of low-dose radiation exposure via induction of defense mechanisms that may improve resilience to hazardous exposures overall (21-24). We believe that all three phenomena might present a complex response to low-dose radiation and these hypotheses are not mutually exclusive. In that case, relative intensity of these responses may differ between individuals; and the predominance of one or a balance between several response types would classify an individual as being extremely sensitive, resistant, or even benefiting from low-dose radiation exposure. On a population level, the risk estimates then would depend on the proportions of highly sensitive and resistant individuals, which in fact, may result in a linear no-threshold relationship with cancer risk (or other health effects) observed statistically. However, biologically such linear relationship may represent a mix of different individual responses within human populations.

It has been argued that the adherence to LNTH is useful as it represents a conservative approach to the risk-benefit analysis (25). However, this might be the case if LNTH is compared to either the threshold or the adaptive response hypotheses. When one considers the bystander effect scenario, reliance in policy-making on the LNTH-based model does not provide adequate protection to individuals with high radiosensitivity. Indeed, enhanced radiosensitivity has been documented among children and individuals with familial cancer predisposition (26-29). Therefore, protection of individuals and groups with high radiosensitivity requires adoption of even more stringent guidelines.

On the other hand, the proponents of the threshold/adaptive responses argue that the existing (conservative) approach has negative economical and societal implications and, therefore, should be relaxed. It was said that LNTH model "imposes excessive costs on the society," "inspires radiophobia" resulting in "refusal of some patients to undergo potentially life-saving medical imaging," and in "discouragement of the studies of low-dose radiation therapies"; moreover, it "provides motivation for radiological terrorism" (30). From either point of view, the LNTH model for radiation risk-benefit analysis seems to be inadequate. 
INDIVIDUALIZED APPROACH TO RADIATION SAFETY AT LOW-DOSE EXPOSURE PROMISES TO AVOID BOTH ENHANCED RISKS AMONG THE RADIOSENSITIVE SUBGROUPS AND AN EXCESSIVE ECONOMIC BURDEN OF AN OVERLY STRICT REGULATIONS

Individualized approach to radiation safety has an immediate practical implication affecting a large part of the general population in the field of medical imaging. The widespread use of computerized tomography (CT) scans has greatly increased public exposure to ionizing radiation (31). It has been estimated that over 70 million/year CT procedures may cause as many as 30,000 new cancer cases in the US alone (32). Because of its superior diagnostic value (33), there is no doubt that the use of CT scans will continue to grow. In this situation, knowing individual radiosensitivity can help to make an informed decision on the risk-benefit ratio in treatment of those who are very sensitive to radiation (34). Similar logic can be applied to other medical and nonmedical situations that involve ionizing radiation exposure. For example, evacuation of residents from areas of low-dose exposure due to radiologic accidents may cause "non-radiogenic disasterrelated premature deaths," such as "officially registered among the evacuated population" in Fukushima (30). Individual approach provides little help in identification of most sensitive individuals after a nuclear catastrophe; however, screening of the population working on and living around nuclear power facilities can inform the urgency of evacuation from the areas with low-dose ionizing radiation (LDIR) exposure. Another example of areas where individualized approach can guide the risk-benefit analysis is testing candidates for spacecraft missions or atomic submarine crews.

\section{INDIVIDUAL VARIABILITY IN RESPONSE TO LOW-DOSE RADIATION HAS BEEN DOCUMENTED}

The phenomenon of individual differences in radiosensitivity has been known since the beginning of the twentieth century (35) and extensively reviewed in previously published reports $(28,36-$ 38). However, the majority of previous studies examined human radiosensitivity to relatively high radiation doses. Regarding LDIR, studies of individual responses are less numerous and inconclusive. Here, we discuss the main advantages and limitations of these studies with the intention to pinpoint specific barriers to establishing population-based research in this area.

It has been suggested that cell and cellular processes are the main targets of the LDIR effects. Naturally, an analysis of individual variability should involve a comparison of cellular responses to LDIR in different individuals. Such a comparison can be conducted using various experimental designs. For example, in vivo biodosimetry studies estimate the frequency of binucleated cells in peripheral blood lymphocytes, comparing individuals with different levels of exposure (39). Biodosimetry studies may help to detect the level of radiation exposure in human population. However, radiation dosimetry may not be appropriate to predict the risk associated with LDIR in un-irradiated population or help to decide whether a substitution of a radiation-based imaging technique should be prescribed to one or another individual. In a more direct manner, individual in vivo response to irradiation was studied by Goldberg et al. (40). Specifically, a small area of skin was irradiated and then a skin biopsy was analyzed for transcriptomic responses. This study yielded two important results: it established that measurable cellular changes occur in the intact human tissues in response to a single low-dose radiation exposure and confirmed the great individual variability of such response. In this setting, individual variability can arise both from genetic predisposition that determines intrinsic radiosensitivity and individual exposures to different factors. For example, differences in diet (41-43), tobacco use $(44,45)$, or prescribed medications (46) can affect individual response (47). Such confounding background factors cannot be easily controlled, compromising comparisons of radiation responses between individuals in vivo. Another disadvantage of the in vivo approach relates to the main goal of such studies, i.e., to predict potential health risks associated with low-dose radiation. The study by Goldberg et al. (40) was conducted among prostate cancer patients scheduled to undergo radiotherapy, i.e., the study population that was to be exposed to higher radiation doses. Obviously, such a direct in vivo radiation test cannot be applied to the general population and especially to individuals suspected to be very sensitive to ionizing radiation.

Alternatively, individual radiosensitivity can be detected using primary cell cultures isolated from different individuals. This approach most importantly evades ethical concerns related to direct irradiation of human subjects. Furthermore, such study design allows a better control of the background factors via standardization of cell culture conditions.

Traditionally, primary cultures of peripheral blood lymphocytes, fibroblasts, or keratinocytes were used as models to study individual response to low-dose radiation $(36,37,48)$. These studies definitely established that even a single very low dose of radiation exposure $(<0.1 \mathrm{~Gy})$ can produced a cellular response. Measurements of the DNA-related effects focused on DNA-damage response $(49,50)$ and alterations in chromatin structure (51). Others studied gene expression $(8,52-56)$, and proteomics (57). Similarly to the studies in vivo, this line of research demonstrated a significant individual variability of cellular responses but has not identified a unified measure of such response that can be used to rank individuals in their radiosensitivity. The main problem with the DNA-targeted and transcriptional responses is their transient nature, which complicates their application to population studies. In addition, these traditional cellular models have limitations either due to low-proliferation potential (primary lymphocytes) or the invasive process of primary cell procurement (as for fibroblasts or keratinocytes).

We would like to note that donor-specific immortalized cell lines also address the concerns related to controlling the background conditions and the ethical concerns of in vivo irradiation. These are good models for studying various cellular functions, except the mechanisms related to cell proliferation control, because immortalization of cells impacts natural mechanisms controlling cell proliferation, and specifically, the signaling pathways responsible for the cell cycle checkpoints. Therefore, donor-specific immortalized cell lines are not suitable for studying potential pro-carcinogenic or anti-carcinogenic responses to low-dose radiation that are related to cell proliferation control. 
To summarize, advances in this research require a new cellular model that satisfies two major requirements: (a) cells can be non-invasively obtained on a population-based scale and (b) produce long-term primary cultures. Such a cellular model would permit search for a non-transient measurable response to low-dose radiation that can rank individuals in their radiosensitivity.

\section{SEARCH FOR A UNIFIED MEASUREMENT TO RANK INDIVIDUALS IN SENSITIVITY TO THE EFFECTS OF LOW-DOSE RADIATION}

The ultimate goal is to develop a test that can be applied to a non-invasively obtained biological sample to assist a medical or a policy decision in risk-benefit analysis, radiation protection, or other scenarios. Application of such test to clinical practice can be similar to the routinely used allergy or antibiotic sensitivity tests. Also, because radiation protection policy often relies on epidemiological data, a radiation sensitivity test would be an important epidemiological tool allowing risk stratification and thereby leading to more precise estimation of risks associated with exposure to low-dose radiation.

This review does not present a comprehensive examination of the existing assays that can be adapted to test individual sensitivity to LDIR. We rather illustrate the concept of cell-based radiation sensitivity testing by presenting several promising approaches. An example could be formation of dicentric chromosomes and gamma-H2AX foci in lymphocytes of human blood samples following exposure to a CT scanner as was shown by Golfier et al. (49). This study found that gamma-H2AX foci formation presents a more sensitive readout as compared to quantification of dicentric chromosomes. However, the transient character of the former would preclude the adoption of such an assay in a populationbased testing; in contrast, the persistence of dicentric chromosome formation may present a more promising readout for the development of a test. The next step would be evaluating the ability of this assay to distinguish individual differences in response to low-dose radiation. Similarly, other measurements related to the cytokinesis-block micronucleus cytome assay in lymphocytes, such as micronuclei (scoring chromosome breakage and/or whole chromosome loss) and nuclear buds (scoring gene amplification) (58) could present the basis for the development of a test, if they prove to be sensitive enough to reveal inter-individual differences. However, it is important to note that these assays are related to DNA-damage effects, whereas LDIR effect on DNA is not substantial. Hence, the development of non-DNA-related cell-based assays is critically needed for testing individual response to LDIR.

In general, the non-DNA-targeted approaches can be classified into three main lines of research and ranked by their ability to produce interpretable information. The "omics" approach including comprehensive analysis of transcriptional and proteome profiles together with dynamic change in metabolome and epigenetics presents a good starting point in cataloging individual responses to LDIR (59). However, the main weakness of this approach is interpretation of the data across different analytical platforms and high variability of results related to assay execution and biospecimen handling conditions (60). As noted by the NCI scientists, so far this approach did not generate data that would clearly lead to the development of any test (60).
Collectively, the "omics" studies suggest that relatively longlived $(>24 \mathrm{~h}$ ) changes in intracellular signaling should be evaluated in LDIR-related cellular responses. Studies focused on the analysis of certain signaling pathways confirmed that LDIR exposure indeed results in long-lived changes in intracellular signaling (60-63). However, a measurable effect that can serve as a biomarker of individual differences in response to low-dose radiation remains to be identified.

Finally, some integrated measurements of cellular functions can lead to a desired outcome. As an example, induction or redirection of cellular differentiation can be studied as an anti- or pro-carcinogenic response, respectively $(64,65)$. This phenomenon has been studied in hematopoietic stem and progenitor cells, which are present at low frequency $(<0.5 \%$ of mononuclear cells) in peripheral and umbilical cord blood. These cells can be identified by the expression of a transmembrane protein CD34 (CD34+ cells) and their differentiation can be followed by appearance of lineage-specific surface cell markers, thus, allowing quantification of cell differentiation response using blood specimens (66). Monzen et al. used purified CD34+ from cord blood to study their differentiation in response to different types and doses of ionizing radiation (66). This study did not find a difference in the total number of mononuclear cells generated in the culture of purified CD34+ cells in response to 0.5-Gy X-ray exposure but the fraction of different lineages has changed: the fraction of cells with eosinophil/neutrophil lineage markers decreased, whereas the erythroid-related lineage fraction increased. This example demonstrates that the analysis of cell differentiation response may present the basis for the development of individual radiosensitivity test.

In summary, cell-based research demonstrated that cellular response to LDIR in vitro can be detected. In vitro donor-specific cellular models have a potential to evolve into an individual radiosensitivity test. However, there is an important caveat: these models and assays should reveal inter-individual variability, be sensitive to detect the response at a low dose, and be amenable to high-throughput screening instrumentation and protocols (67).

\section{AT THE INTERSECTION OF EPIDEMIOLOGY AND CELL BIOLOGY: OUR EXPERIENCE WITH A MODEL FOR INDIVIDUAL RESPONSE TO LDIR}

An epidemiological cell-based model should (a) be donor-specific, (b) allow for robust in vitro culturing, thus, amenable for highthroughput screening, (c) produce highly viable cultures after cryopreservation, and (d) be obtained in a least invasive manner.

Blood-derived CD31+/CD34+ endothelial colony-forming cells (ECFCs) were found to fulfill these requirements. ECFCs form highly proliferative cell cultures originating from a single cell, which forms a colony of progenitors (hence, the name) under certain growth conditions. These cells can be propagated over many passages maintaining endothelial phenotype (68). We found that ECFCs can be cryopreserved for many months and even years. Therefore, we isolated ECFCs from cord blood of three donors to search for quantifiable cellular responses to low-dose radiation (69). We found that a single radiation dose of 0.05 Gy significantly inhibits cell proliferation, a response that is not observed in immortalized cell lines at such a low dose. The response appears to be donor-specific (69). Importantly, this LDIR effect appeared 
only $48 \mathrm{~h}$ post-irradiation. We have recently extended our observation to even lower dose of $0.01 \mathrm{~Gy}$ (data not published). We did not find any indication of ECFCs' death after irradiation. Thus, LDIR inhibits ECFCs proliferation but does not kill the cells suggesting that such a treatment promotes either cell senescence or differentiation.

Our experience working with donor-specific ECFCs as a model lead to several important conclusions. First, these cells are very sensitive to low-dose radiation. Second, non-DNA-based readouts such as proliferation can be used to analyze response to LDIR because they can persist for several days. Third, the effects of LDIR can be quantified in cell cultures that are donor-specific, thus, providing the bases for individual testing. Although this research has been only recently initialized and is still at its infancy, it indicates that donor-specific radiation sensitivity testing is achievable.

\section{CONCLUSION}

The LNT model of the health risk associated with low-dose radiation has been criticized on the basis of different biological effects induced by high- vs. low-doses of radiation. However, whether the departure from linearity is toward increased or decreased risk presents a hot point of the debate in the field of radiation riskbenefit analysis and radiation protection. We hypothesize that both increased and decreased risks are possible depending on the individual response to LDIR. In fact, there is a consensus that individuals differ in their radiosensitivity. Although it has been recognized that the main effects of LDIR are not related to DNA damage per se, the initial search for indicators of individual radiosensitivity has been rooted in quantification of DNA-damage responses. The low levels and the transitory nature of DNA-damage related indicators demonstrated the futility of this approach. The next phase in this research addressed the question of which genes, proteins, and pathways are responsible for specific responses to LDIR. This phase entailed screening different "omics" platforms to catalog biological responses induced by LDIR and confirmed first, that many effects are transitory and second, that individual responses vary indeed. However, these approaches could not capture quantifiable indicators of individual responses. Currently, this search is focused on cellular models that can be donor-specific and satisfy the noninvasive requirement of cell procurement. We share our experience working with blood-derived donor-specific ECFCs as a model to find an indicator of individual radiosensitivity. Other assays using blood-derived cells confirm that this can be a promising approach. The goal now is to find a quantifiable measure of cellular response amiable to high-throughput population testing that is sensitive enough to rank individuals by their radiosensitivity.

\section{REFERENCES}

1. United Nations Scientific Committee on the Effects of Atomic Radiation. UNSCEAR 2006 Report to the General Assembly with Scientific Annexes. Sources and Effects of Ionizing Radiation. Volume I Report and Annexes A and B. New York, NY: United Nations (2008).

2. IARC. Radiation. IARC Monographs on The Evaluation of Carcinogenic Risks to Humans. World Health Organization, International Agency for Research on Cancer. (2012) 100(Pt D):7-303.

3. Preston RJ, Boice JD Jr., Brill AB, Chakraborty R, Conolly R, Hoffman FO, et al. Uncertainties in estimating health risks associated with exposure to ionising radiation. J Radiol Prot (2013) 33(3):573-88. doi:10.1088/0952-4746/ $33 / 3 / 573$
4. Dauer LT, Brooks AL, Hoel DG, Morgan WF, Stram D, Tran P, et al. Review and evaluation of updated research on the health effects associated with low-dose ionising radiation. Radiat Prot Dosimetry (2010) 140(2):103-36. doi: $10.1093 / \mathrm{rpd} / \mathrm{ncq} 141$

5. Feinendegen LE, Brooks AL, Morgan WF. Biological consequences and health risks of low-level exposure to ionizing radiation: commentary on the workshop. Health Phys (2011) 100(3):247-59. doi:10.1097/HP.0b013e31820a83ae

6. Tubiana M, Feinendegen LE, Yang C, Kaminski JM. The linear no-threshold relationship is inconsistent with radiation biologic and experimental data. Radiology (2009) 251(1):13-22. doi:10.1148/radiol.2511080671

7. Averbeck D. Non-targeted effects as a paradigm breaking evidence. Mutat Res (2010) 687(1-2):7-12. doi:10.1016/j.mrfmmm.2010.01.004

8. Ray M, Yunis R, Chen X, Rocke DM. Comparison of low and high dose ionising radiation using topological analysis of gene coexpression networks. BMC Genomics (2012) 13:190. doi:10.1186/1471-2164-13-190

9. Morgan WF. Non-targeted and delayed effects of exposure to ionizing radiation: I. Radiation-induced genomic instability and bystander effects in vitro. Radiat Res (2003) 159(5):567-80. doi:10.1667/0033-7587(2003) 159[0581:NADEOE]2.0.CO;2

10. Morgan WF. Non-targeted and delayed effects of exposure to ionizing radiation: II. Radiation-induced genomic instability and bystander effects in vivo, clastogenic factors and transgenerational effects. Radiat Res (2003) 159(5):581-96. doi:10.1667/0033-7587(2003)159[0581:NADEOE]2.0.CO;2

11. Yang F, Stenoien DL, Strittmatter EF, Wang J, Ding L, Lipton MS, et al. Phosphoproteome profiling of human skin fibroblast cells in response to low- and highdose irradiation. J Proteome Res (2006) 5(5):1252-60. doi:10.1021/pr060028v

12. Amundson SA, Fornace AJ Jr. Monitoring human radiation exposure by gene expression profiling: possibilities and pitfalls. Health Phys (2003) 85(1):36-42. doi:10.1097/00004032-200307000-00009

13. Blimkie MSJ, Fung LCW, Petoukhov ES, Girard C, Klokov D. Repair of DNA double-strand breaks is not modulated by low-dose gamma radiation in C57BL/6J mice. Radiat Res (2014) 181(5):548-59. doi:10.1667/RR13324.1

14. U.S. National Academy of Sciences NRC. Committee to Assess Health Risks from Exposure to Low Levels of Ionizing Radiation. Health Risks from Exposure to Low Levels of Ionizing Radiation: BEIR VII - Phase 2. Washington, DC: National Academies of Science, Center for Education (2004). 56 p.

15. The 2007 Recommendations of the International Commission on Radiological Protection. ICRP publication 103. Ann ICRP (2007) 37(2-4):1-332.

16. Little MP, Wakeford R, Tawn EJ, Bouffler SD, Berrington de Gonzalez A. Risks associated with low doses and low dose rates of ionizing radiation: why linearity may be (almost) the best we can do. Radiology (2009) 251(1):6-12. doi:10.1148/radiol.2511081686

17. Brenner DJ. Is the linear-no-threshold hypothesis appropriate for use in radiation protection? Favouring the proposition. Radiat Prot Dosimetry (2001) 97(3):279-82. doi:10.1093/oxfordjournals.rpd.a006675

18. Mothersill C, Seymour CB. Radiation-induced bystander effects - implications for cancer. Nat Rev Cancer (2004) 4(2):158-64. doi:10.1038/nrc1277

19. Wright EG. Manifestations and mechanisms of non-targeted effects of ionizing radiation. Mutat Res (2010) 687(1/2):28-33. doi:10.1016/j.mrfmmm.2010. 01.014

20. Scott BR. Stochastic thresholds: a novel explanation of nonlinear doseresponse relationships for stochastic radiobiological effects. Dose Response (2006) 3(4):547-67. doi:10.2203/dose-response.003.04.009

21. Sasaki MS, Tachibana A, Takeda S. Cancer risk at low doses of ionizing radiation: artificial neural networks inference from atomic bomb survivors. J Radiat Res (2014) 55(3):391-406. doi:10.1093/jrr/rrt133

22. Tapio S, Jacob V. Radioadaptive response revisited. Radiat Environ Biophys (2007) 46(1):1-12. doi:10.1007/s00411-006-0078-8

23. Sanders CL. Potential treatment of inflammatory and proliferative diseases by ultra-low doses of ionizing radiations. Dose Response (2012) 10(4):610-25. doi:10.2203/dose-response.12-017.Sanders

24. Doss M. Linear no-threshold model vs. radiation hormesis. Dose Response (2013) 11:480-97. doi:10.2203/dose-response.13-005.Doss

25. Puskin JS. Perspective on the use of LNT for radiation protection and risk assessment by the U.S. Environmental Protection Agency. Dose Response (2009) 7(4):284-91. doi:10.2203/dose-response.09-005.Puskin

26. Wakeford R, Little MP. Risk coefficients for childhood cancer after intrauterine irradiation: a review. Int J Radiat Biol (2003) 79(5):293-309. doi:10.1080/ 0955300031000114729 
27. Roberts SA, Spreadborough AR, Bulman B, Barber JB, Evans DG, Scott D. Heritability of cellular radiosensitivity: a marker of low-penetrance predisposition genes in breast cancer? Am J Hum Genet (1999) 65(3):784-94. doi:10.1086/ 302544

28. Ronckers CM, Doody MM, Lonstein JE, Stovall M, Land CE. Multiple diagnostic X-rays for spine deformities and risk of breast cancer. Cancer Epidemiol Biomarkers Prev (2008) 17(3):605-13. doi:10.1158/1055-9965.EPI-07-2628

29. Davis F, Il'yasova D, Rankin K, McCarthy B, Bigner DD. Medical diagnostic radiation exposures and risk of gliomas. Radiat Res (2011) 175(6):790-6. doi:10.1667/RR2186.1

30. Socol Y, Dobrzynski L, Doss M, Feinendegen LE, Janiak MK, Miller ML, et al. Commentary: ethical issues of current health-protection policies on lowdose ionizing radiation. Dose Response (2013) 12(2):342-8. doi:10.2203/doseresponse.13-044

31. González ABD, Kim K, Knudsen AB, Lansdorp-Vogelaar I, Rutter CM, SmithBindman R, et al. Radiation-related cancer risks from CT colonography screening: a risk-benefit analysis. Am J Roentgenol (2011) 196(4):816-23. doi:10.2214/ AJR.10.4907

32. González ABD, Mahesh M, Kwang-Pyo K, Bhargavan M, Lewis R, Mettler F, et al. Projected cancer risks from computed tomographic scans performed in the United States in 2007. Arch Intern Med (2009) 169(22):2071-7. doi:10.1001/archinternmed.2009.440

33. Amis ES Jr., Butler PF, Applegate KE, Birnbaum SB, Brateman LF, Hevezi JM, et al. American College of Radiology white paper on radiation dose in medicine. J Am Coll Radiol (2007) 4(5):272-84. doi:10.1016/j.jacr.2007.03.002

34. Bukowski JA, Lewis RJ. Practical implications of nonlinear effects in riskassessment harmonization. Nonlinearity Biol Toxicol Med (2004) 2(1):3-10. doi:10.1080/15401420490426927

35. Vogin G, Foray N. The law of Bergonié and Tribondeau: a nice formula for a first approximation. Int J Radiat Biol (2013) 89(1):2-8. doi:10.3109/09553002. 2012.717732

36. Kato TA, Wilson PF, Nagasaw H, Peng Y, Weil MM, Little JB, et al. Variations in radiosensitivity among individuals: a potential impact on risk assessment? Health Phys (2009) 97(5):470-80. doi:10.1097/HP.0b013e3181b08eee

37. Maalouf M, Durante M, Foray N. Biological effects of space radiation on human cells: history, advances and outcomes. J Radiat Res (2011) 52(2):126-46. doi:10.1269/jrr.10128

38. Greve B, Bölling T, Amler S, Rössler U, Gomolka M, Mayer C, et al. Evaluation of different biomarkers to predict individual radiosensitivity in an inter-laboratory comparison - lessons for future studies. PLoS One (2012) 7(10):e47185. doi:10.1371/journal.pone.0047185

39. Tucker JD, Vadapalli M, Joiner MC, Ceppi M, Fenech M, Bonassi S. Estimating the lowest detectable dose of ionizing radiation by the cytokinesis-block micronucleus assay. Radiat Res (2013) 180(3):284-91. doi:10.1667/RR3346.1

40. Goldberg Z, Rocke DM, Schwietert C, Berglund SR, Santana A, Jones A, et al. Human in vivo dose-response to controlled, low-dose low linear energy transfer ionizing radiation exposure. Clin Cancer Res (2006) 12(12):3723-9. doi:10.1158/1078-0432.CCR-05-2625

41. Morgan JL, Ritchie LE, Crucian BE, Theriot C, Wu HL, Sams C, et al. Increased dietary iron and radiation in rats promote oxidative stress, induce localized and systemic immune system responses, and alter colon mucosal environment. FASEB J (2014) 28(3):1486-98. doi:10.1096/fj.13-239418

42. Batra V, Kislay B. Mitigation of gamma-radiation induced abasic sites in genomic DNA by dietary nicotinamide supplementation: metabolic up-regulation of NAD+ biosynthesis. Mutat Res (2013) 749(1/2):28-38. doi:10.1016/j.mrfmmm. 2013.07.001

43. Mortazavi SMJ. Space radiobiology and the new era of induced radioresistance: should traditional concepts be moved to science history museums? Technol Health Care (2013) 21(4):285-9. doi:10.3233/THC- 130732

44. Rugg T, Saunders MI, Dische S. Smoking and mucosal reactions to radiotherapy. Br J Radiol (1990) 63(751):554-6. doi:10.1259/0007-1285-63-751-554

45. Browman GP, Mohide EA, Willan A, Hodson I, Wong G, Grimard L, et al. Association between smoking during radiotherapy and prognosis in head and neck cancer: a follow-up study. Head Neck (2002) 24(12):1031-7. doi:10.1002/hed. 10168

46. Biju PG, Gubrij I, Garg S, Gupta PK, Hauer-Jensen M, Burnett AF. Radiation lethality potentiation in total body irradiated mice by a commonly prescribed proton pump inhibitor, pantoprazole sodium. Int J Radiat Biol (2014) 90(7):554-9. doi:10.3109/09553002.2014.905727
47. Nenoi M, Wang B, Vares G. In vivo radioadaptive response: a review of studies relevant to radiation-induced cancer risk. Hum Exp Toxicol (2014). doi:10.1177/0960327114537537

48. Story M, Ding LH, Brock WA, Ang KK, Alsbeih G, Minna J, et al. Defining molecular and cellular responses after low and high linear energy transfer radiations to develop biomarkers of carcinogenic risk or therapeutic outcome. Health Phys (2012) 103(5):596-606. doi:10.1097/HP.0b013e3182692085

49. Golfier S, Jost G, Pietsch H, Lengsfeld P, Eckardt-Schupp F, Schmid E, et al. Dicentric chromosomes and gamma-H2AX foci formation in lymphocytes of human blood samples exposed to a CT scanner: a direct comparison of dose response relationships. Radiat Prot Dosimetry (2009) 134(1):55-61. doi:10.1093/rpd/ncp061

50. Wilson PF, Nham PB, Urbin SS, Hinz JM, Jones IM, Thompson LH. Interindividual variation in DNA double-strand break repair in human fibroblasts before and after exposure to low doses of ionizing radiation. Mutat Res (2010) 683(1-2):91-7. doi:10.1016/j.mrfmmm.2009.10.013

51. Jain V, Hassan PA, Das B. Radiation-induced conformational changes in chromatin structure in resting human peripheral blood mononuclear cells. Int J Radiat Biol (2014):1-9. doi:10.3109/09553002.2014.925605

52. Knops K, Boldt S, Wolkenhauer O, Kriehuber R. Gene expression in lowand high-dose-irradiated human peripheral blood lymphocytes: possible applications for biodosimetry. Radiat Res (2012) 178(4):304-12. doi:10.1667/ RR2913.1

53. Turtoi A, Brown I, Oskamp D, Schneeweiss FHA. Early gene expression in human lymphocytes after gamma-irradiation-a genetic pattern with potential for biodosimetry. Int J Radiat Biol (2008) 84(5):375-87. doi:10.1080/ 09553000802029886

54. Manning G, Kabacik S, Finnon P, Bouffler S, Badie C. High and low dose responses of transcriptional biomarkers in ex vivo X-irradiated human blood. Int J Radiat Biol (2013) 89(7):512-22. doi:10.3109/09553002.2013.769694

55. Ding LH, Shingyoji M, Chen F, Hwang J-J, Burma S, Lee C, et al. Gene expression profiles of normal human fibroblasts after exposure to ionizing radiation: a comparative study of low and high doses. Radiat Res (2005) 164(1):17-26. doi:10.1667/RR3354

56. Franco N, Lamartine J, Frouin V, Le Minter P, Petat C, Leplat JJ, et al. Low-dose exposure to gamma rays induces specific gene regulations in normal human keratinocytes. Radiat Res (2005) 163(6):623-35. doi:10.1667/RR3391

57. Tapio S. Ionizing radiation effects on cells, organelles and tissues on proteome level. Adv Exp Med Biol (2013) 990:37-48. doi:10.1007/978-94-007-5896-4_2

58. Thomas P, Fenech M. Cytokinesis-block micronucleus cytome assay in lymphocytes. Methods Mol Biol (2011) 682:217-34. doi:10.1007/978-1-60327409-8_16

59. Shay JW, Cucinotta FA, Sulzman FM, Coleman CN, Minna JD. From mice and men to earth and space: joint NASA-NCI workshop on lung cancer risk resulting from space and terrestrial radiation. Cancer Res (2011) 71(22):6926-9. doi:10.1158/0008-5472.CAN-11-2546

60. NCI issues omics checklist for tests. Cancer Discov (2014) 4(2):OF6. doi:10. 1158/2159-8290.CD-NB2013-157

61. Klokov D, Leskov K, Araki S, Zou Y, Goetz EM, Luo X, et al. Low dose IR-induced IGF-1-sCLU expression: a p53-repressed expression cascade that interferes with TGF $\beta 1$ signaling to confer a pro-survival bystander effect. Oncogene (2013) 32(4):479-90. doi:10.1038/onc.2012.64

62. Mariotti LG, Bertolotti A, Ranza E, Babini G, Ottolenghi A. Investigation of the mechanisms underpinning IL-6 cytokine release in bystander responses: the roles of radiation dose, radiation quality and specific ROS/RNS scavengers. Int J Radiat Biol (2012) 88(10):751-62. doi:10.3109/09553002.2012.703365

63. Zhang Q, Matzke M, Schepmoes AA, Moore RJ, Webb-Robertson BJ, Hu Z, et al. High and low doses of ionizing radiation induce different secretome profiles in a human skin model. PLoS One (2014) 9(3):e92332. doi:10.1371/journal.pone. 0092332

64. Klug F, Prakash H, Huber PE, Seibel T, Bender N, Halama N, et al. Low-dose irradiation programs macrophage differentiation to an iNOS+/M1 phenotype that orchestrates effective T cell immunotherapy. Cancer Cell (2013) 24(5):589-602. doi:10.1016/j.ccr.2013.09.014

65. Floor SL, Dumont JE, Maenhaut C, Raspe E. Hallmarks of cancer: of all cancer cells, all the time? Trends Mol Med (2012) 18(9):509-15. doi:10.1016/j.molmed. 2012.06.005

66. Monzen S, Yoshino H, Kasai-Eguchi K, Kashiwakura I. Characteristics of myeloid differentiation and maturation pathway derived from human hematopoietic 
stem cells exposed to different linear energy transfer radiation types. PLoS One (2013) 8(3):e59385. doi:10.1371/journal.pone.0059385

67. Brenner DJ. Exploring two two-edged swords. Radiat Res (2012) 178(1):7-16. doi:10.1667/RR3085.1

68. Huang L, Critser PJ, Grimes BR, Yoder MC. Human umbilical cord blood plasma can replace fetal bovine serum for in vitro expansion of functional human endothelial colony-forming cells. Cytotherapy (2011) 13(6):712-21. doi:10.3109/14653249.2010.548380

69. Kinev AV, Levering V, Young K, Ali-Osman F, Truskey GA, Dewhirst MW, et al. Endothelial colony forming cells (ECFCs) as a model for studying effects of low-dose ionizing radiation: growth inhibition by a single dose. Cancer Invest (2013) 31(5):359-64. doi:10.3109/07357907.2013.789903

Conflict of Interest Statement: Alexander Kinev is a founder and executive of Creative Scientist, Inc. He is also a majority shareholder of this company, which develops a radiation sensitivity test. No other conflicts of interest are reported. This research was supported by an epidemiology grant from the National Brain Tumor Society.

Received: 01 October 2014; accepted: 05 November 2014; published online: 18 November 2014.

Citation: Il'yasova D, Kinev A, Melton CD and Davis FG (2014) Donor-specific cell-based assays in studying sensitivity to low-dose radiation: a population-based perspective. Front. Public Health 2:244. doi: 10.3389/fpubh.2014.00244

This article was submitted to Epidemiology, a section of the journal Frontiers in Public Health.

Copyright $\odot 2014$ Il'yasova, Kinev, Melton and Davis. This is an open-access article distributed under the terms of the Creative Commons Attribution License (CC BY). The use, distribution or reproduction in other forums is permitted, provided the original author(s) or licensor are credited and that the original publication in this journal is cited, in accordance with accepted academic practice. No use, distribution or reproduction is permitted which does not comply with these terms. 